\title{
The Rearrangement of Dicarboranyl Methyl Cation: A Possible Synthetic Strategy Towards Cationic Closo-Tricarbaboranes
}

\author{
Eluvathingal D. Jemmis, ${ }^{*}$ Elambalassery G. Jayasree \\ School of Chemistry, University of Hyderabad, Hyderabad-500046, India. \\ e-mail: jemmis@uohyd.ernet.in
}

\section{Supplementary Information}

Scheme 1. The benzyl-tropylium ion equivalent equilibria computed for the monocarbaboranes. The optimized geometry of the reactants (Bz), transition states (TS), and the products ( $T r$ ); the energy barrier, Ea $\left(\mathrm{kcal} \mathrm{mol}^{-1}\right)$; and the relative energies, RE $\left(\mathrm{kcal} \mathrm{mol}^{-1}\right)$ at $\mathrm{B} 3 \mathrm{LYP} / 6-31 \mathrm{G}^{*}$ are given. TS is characterized with one imaginary vibrational frequency.

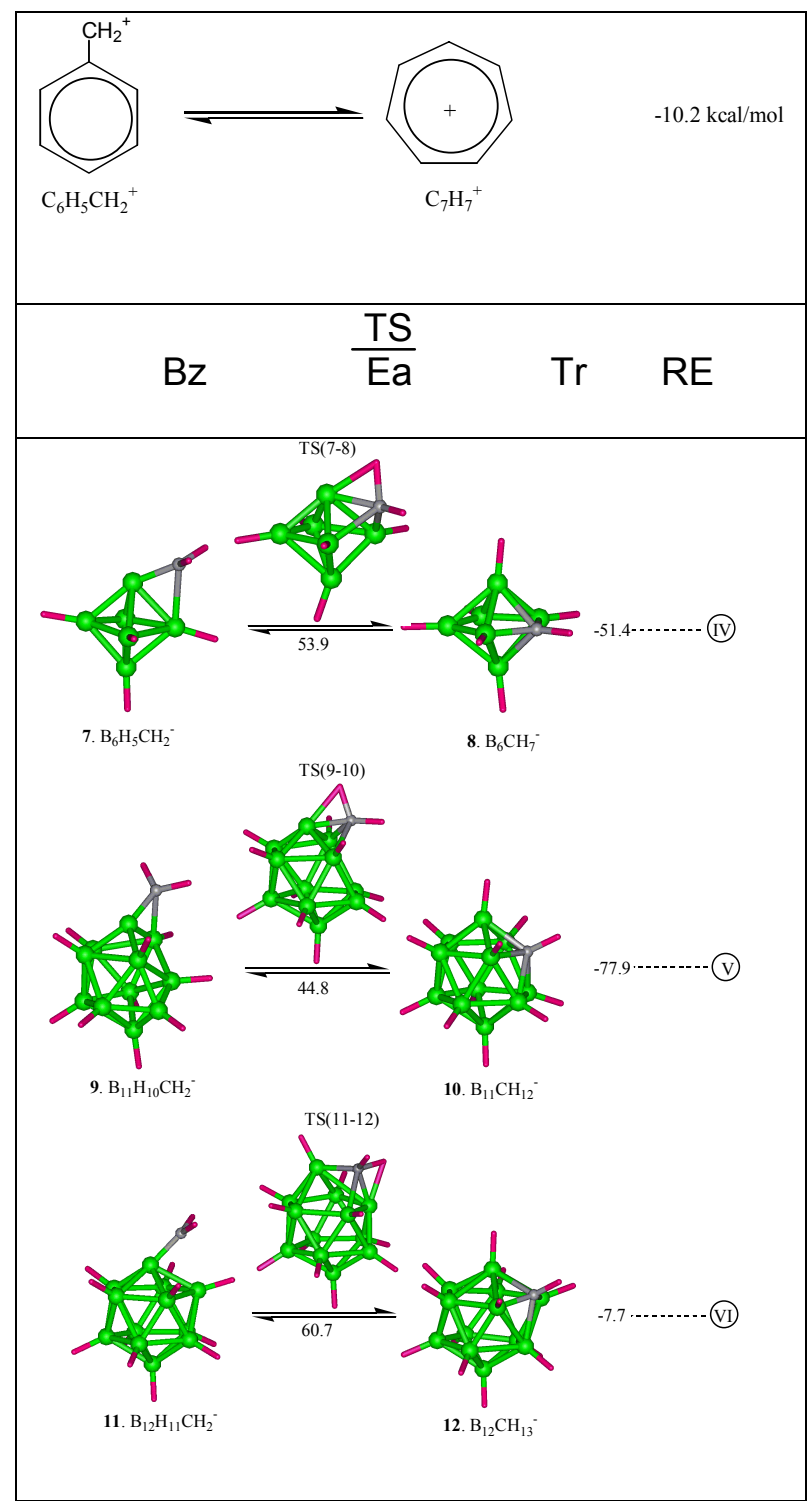


Figure 1: The transition states (TS(13-14) and TS(15-14)) and the energy barriers obtained for the formation of the icosahedral cation (14) starting from the experimentally known 11-vertex dicarborane with $\mathrm{CH}_{2}$ substituted on boron, 13 as well as on carbon vertex, 15.

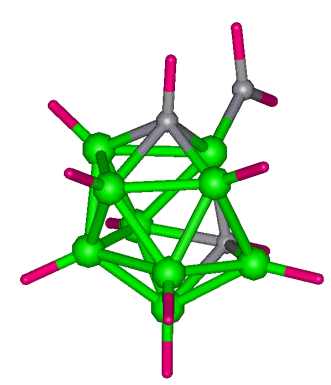

13. $2-\mathrm{CH}_{2}, 1,7-\mathrm{C}_{2} \mathrm{~B}_{9} \mathrm{H}_{10}$
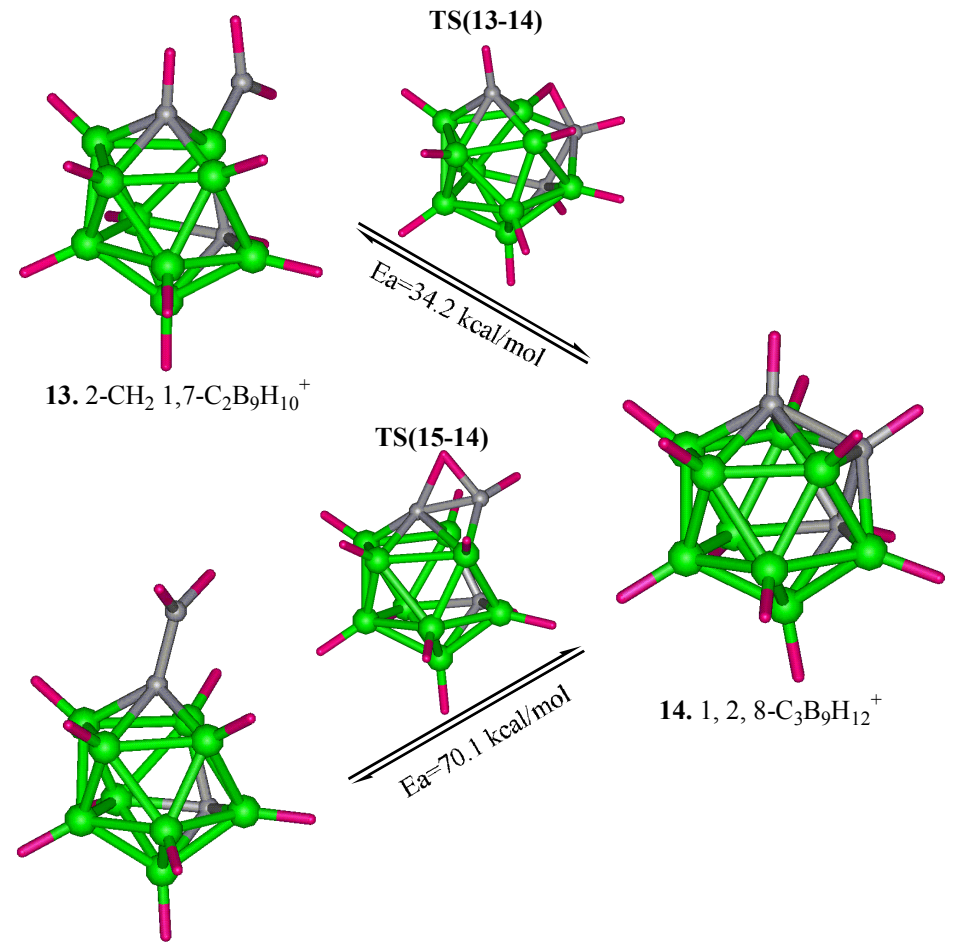

14. $1,2,8-\mathrm{C}_{3} \mathrm{~B}_{9} \mathrm{H}_{12}{ }^{+}$

15. $1-\mathrm{CH}_{2} 1,7-\mathrm{C}_{2} \mathrm{~B}_{9} \mathrm{H}_{10}{ }^{+}$

Figure 2: The optimized geometries of anionic dicarboranyl methyl systems with planar methyl carbon and trianionic boranly methyl systems with pyramidal methyl cabron. In the dicarboranyl methyl systems the cage, due to its electron withdrawing character, incorporates the additional electron pair for the skeletal bonding and opens up. The optimized structures are in accord with the fact that the methyl anion is pyramidal whereas the methyl cation takes on a planar arrangement.

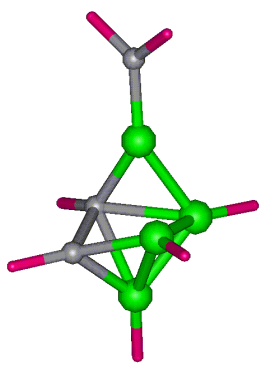

$1-\mathrm{CH}_{2} 2,3-\mathrm{C}_{2} \mathrm{~B}_{4} \mathrm{CH}_{2}^{-}$

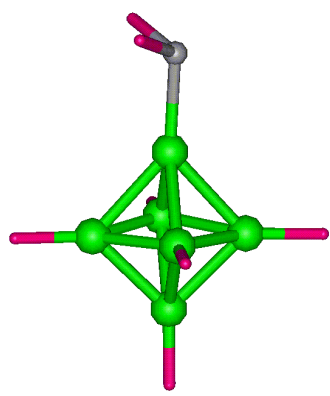

$\mathrm{B}_{6} \mathrm{H}_{5} \mathrm{CH}_{2}^{-3}$

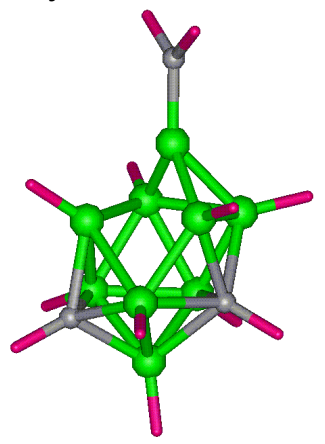

$1-\mathrm{CH}_{2} 7,9-\mathrm{C}_{2} \mathrm{~B}_{9} \mathrm{H}_{10}{ }^{-}$

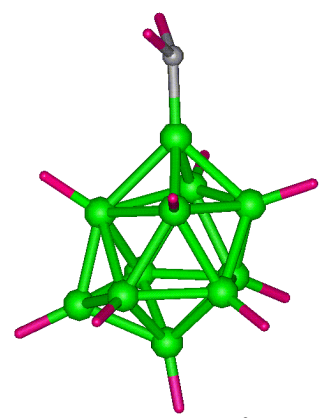

$\mathrm{B}_{11} \mathrm{H}_{10} \mathrm{CH}_{2}^{-3}$

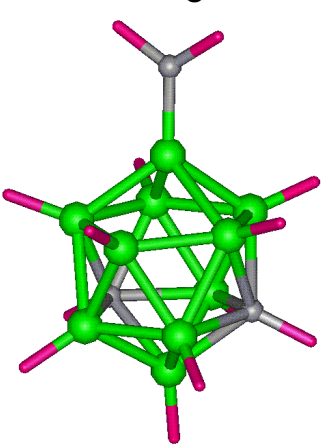

$1-\mathrm{CH}_{2} 8,10-\mathrm{C}_{2} \mathrm{~B}_{10} \mathrm{H}_{11}$

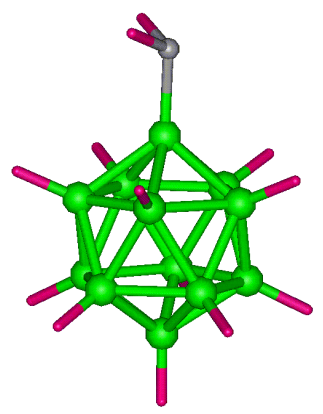

$\mathrm{B}_{12} \mathrm{H}_{11} \mathrm{CH}_{2}^{-3}$ 
Table 1: Total Energies(au) of all the structures calculated at the B3LYP/6-31G* level of theory.

\begin{tabular}{|c|c|}
\hline Compound & Energy, au \\
\hline $1-\mathrm{CH}_{2} 2,3-\mathrm{C}_{2} \mathrm{~B}_{4} \mathrm{H}_{5}^{+}(1)$ & -217.60895 \\
\hline TS(1-2) & -217.52663 \\
\hline $2,4,5-\mathrm{C}_{3} \mathrm{~B}_{4} \mathrm{H}_{7}^{+}(2)^{[2 \mathrm{a}]}$ & -217.69499 \\
\hline $1-\mathrm{CH}_{2} 7,9-\mathrm{C}_{2} \mathrm{~B}_{9} \mathrm{H}_{10}{ }^{+}(3)$ & -345.00457 \\
\hline TS(3-4) & -344.95797 \\
\hline $2,8,10-\mathrm{C}_{3} \mathrm{~B}_{9} \mathrm{H}_{12}{ }^{+}(4)^{[2 \mathrm{a}]}$ & -345.07043 \\
\hline $1-\mathrm{CH}_{2} 8,10-\mathrm{C}_{2} \mathrm{~B}_{10} \mathrm{H}_{11}{ }^{+}(5)$ & -370.50948 \\
\hline TS(5-6) & -370.40212 \\
\hline $2,9,11-\mathrm{C}_{3} \mathrm{~B}_{10} \mathrm{H}_{13}{ }^{+}(\mathbf{6})$ & -370.49273 \\
\hline $\mathrm{B}_{6} \mathrm{H}_{5} \mathrm{CH}_{2}^{-}(7)$ & -191.45219 \\
\hline TS(7-8) & -191.36634 \\
\hline $\mathrm{B}_{6} \mathrm{CH}_{7}^{-}(8)$ & -191.53405 \\
\hline $\mathrm{B}_{11} \mathrm{H}_{10} \mathrm{CH}_{2}^{-}(\mathbf{9})$ & -318.87012 \\
\hline TS $(9-10)$ & -318.79878 \\
\hline $\mathrm{B}_{11} \mathrm{CH}_{12}(\mathbf{1 0})$ & -318.99429 \\
\hline $\mathrm{B}_{12} \mathrm{H}_{11} \mathrm{CH}_{2}^{-}(11)$ & -344.39207 \\
\hline $\mathrm{TS}(11-12)$ & -344.29530 \\
\hline $\mathrm{B}_{12} \mathrm{CH}_{13}^{-}(\mathbf{1 2})$ & -344.40427 \\
\hline $2-\mathrm{CH}_{2}-1,7-\mathrm{C}_{2} \mathrm{~B}_{9} \mathrm{H}_{10}{ }^{+}(13)$ & -344.97573 \\
\hline $\mathrm{TS}(13-14)$ & -344.92119 \\
\hline $1,2,8-\mathrm{C}_{3} \mathrm{~B}_{9} \mathrm{H}_{12}{ }^{+}(\mathbf{1 4})^{[2 \mathrm{a}]}$ & -345.03934 \\
\hline $1-\mathrm{CH}_{2}-1,7-\mathrm{C}_{2} \mathrm{~B}_{9} \mathrm{H}_{10}{ }^{+}(15)$ & -344.98245 \\
\hline $\mathrm{TS}(15-14)$ & -344.87076 \\
\hline $1-\mathrm{CH}_{2} 2,3-\mathrm{C}_{2} \mathrm{~B}_{4} \mathrm{H}_{5}^{-}$ & -217.90770 \\
\hline $1-\mathrm{CH}_{2} 7,9-\mathrm{C}_{2} \mathrm{~B}_{9} \mathrm{H}_{10^{-}}$ & -345.29720 \\
\hline $1-\mathrm{CH}_{2} 8,10-\mathrm{C}_{2} \mathrm{~B}_{10} \mathrm{H}_{11}$ & -370.78891 \\
\hline $\mathrm{B}_{6} \mathrm{H}_{5} \mathrm{CH}_{2}^{-3}$ & -190.96884 \\
\hline $\mathrm{B}_{11} \mathrm{H}_{10} \mathrm{CH}_{2}^{-3}$ & -318.48822 \\
\hline $\mathrm{B}_{12} \mathrm{H}_{11} \mathrm{CH}_{2}^{-3}$ & -344.05675 \\
\hline
\end{tabular}


The optimized coordinates of the structures calculated at B3LYP/6-31G* level of theory

$\begin{array}{lrrr}1-\mathrm{CH}_{2} & 2,3-\mathrm{C}_{2} \mathrm{~B}_{4} \mathrm{H}_{5}{ }^{+}(\mathbf{1}) \\ 6 & -0.383337 & 0.873903 & 0.812042 \\ 1 & -2.754564 & 0.237742 & 0.000000 \\ 5 & -0.383337 & -0.739563 & -0.898310 \\ 6 & -0.383337 & 0.873903 & -0.812042 \\ 5 & -0.383337 & -0.739563 & 0.898310 \\ 5 & -1.582165 & 0.135349 & 0.000000 \\ 5 & 0.849387 & 0.310947 & 0.000000 \\ 1 & -0.440884 & 1.735643 & 1.466096 \\ 6 & 1.943579 & -0.650439 & 0.000000 \\ 1 & -0.302654 & -1.481636 & -1.806706 \\ 1 & -0.302654 & -1.481636 & 1.806706 \\ 1 & -0.440884 & 1.735643 & -1.466096 \\ 1 & 2.338735 & -1.082908 & 0.918053 \\ 1 & 2.338735 & -1.082908 & -0.918053\end{array}$

\section{$\mathrm{TS}(1-2)$}

1- $\mathrm{CH}_{2} 7,9-\mathrm{C}_{2} \mathrm{~B}_{9} \mathrm{H}_{10}{ }^{+}(3)$

$\begin{array}{lrrr}5 & -0.119675 & -0.036172 & 1.557179 \\ 5 & -1.643073 & 0.394047 & -0.831348 \\ 5 & 1.418070 & 0.531106 & -0.821670 \\ 5 & -0.108230 & 1.338885 & -0.896802 \\ 5 & -0.061321 & -0.501917 & -1.339113 \\ 5 & -1.682739 & -0.432950 & 0.795181 \\ 5 & 1.239996 & -0.419012 & 0.569947 \\ 6 & -0.960546 & 1.123398 & 0.575813 \\ 6 & -1.344230 & -1.151762 & -0.629816 \\ 5 & -0.255717 & -1.491445 & 0.463024 \\ 5 & 0.681580 & 1.304872 & 0.695402 \\ 1 & -2.562775 & 0.842979 & -1.415241 \\ 1 & 2.155836 & 0.975227 & -1.633226 \\ 1 & -0.225764 & 2.300515 & -1.572496 \\ 1 & 0.283723 & -0.994291 & -2.359952 \\ 1 & -2.662631 & -0.600934 & 1.426861 \\ 6 & 2.599589 & -0.645779 & 0.078761 \\ 1 & -1.523219 & 1.947332 & 1.004050\end{array}$
$-1.544633$
$-0.054038$
$-0.089425$
$-0.292631$
$-0.803796$
0.828482
$-0.626107-1.688963$
$\begin{array}{ll}1.825142 & -1.521322\end{array}$
1.532538
$-2.485148-0.500915$
$-0.437242$
$-0.568382$
1.106069
0.386894
$-0.788716$
2.208981
0.755775
0.748932
0.714623
$-0.679417$
0.957775
$-0.890895$
$-0.289207$
1.367209
1.300471
$-1.349682$
$-0.296872$
$-0.439077$
$-0.965829$
$-1.620432$
$-0.101105$
1.534254
$-1.334991$
0.836378

$\begin{array}{lrrr}1 & -1.947256 & -1.921368 & -1.096469 \\ 1 & -0.018126 & -2.612072 & 0.753887 \\ 1 & 1.174378 & 2.221375 & 1.253366 \\ 1 & -0.089735 & -0.051634 & 2.738724 \\ 1 & 3.440732 & -0.011596 & 0.350990 \\ 1 & 2.861500 & -1.487741 & -0.558041\end{array}$

\section{$\mathrm{TS}(3-4)$}

$\begin{array}{rrrr}5 & 1.260886 & 0.886280 & -0.769467 \\ 5 & 0.372296 & 1.498004 & 0.763711 \\ 5 & -0.106661 & -0.051283 & 1.730350 \\ 5 & -1.282119 & -0.909786 & 0.683207 \\ 1 & 0.551360 & 2.543067 & 1.281399 \\ 1 & 2.262761 & -1.422073 & -0.874484 \\ 5 & 1.311176 & 0.097665 & 0.856967 \\ 1 & 2.045504 & 1.689427 & -1.147352 \\ 5 & -0.108064 & -0.119065 & -1.695566 \\ 1 & 2.801943 & -0.335020 & 0.466415 \\ 6 & 1.918995 & -0.578189 & -0.287841 \\ 1 & -0.744761 & 2.412833 & -1.286636 \\ 1 & 0.020442 & -0.229094 & -2.866159 \\ 5 & -0.470086 & 1.344810 & -0.862505 \\ 6 & -0.592878 & -1.313383 & -0.742412 \\ 5 & -1.617772 & 0.025858 & -0.776019 \\ 5 & 0.480556 & -1.432875 & 0.552378 \\ 1 & -2.105287 & -1.531534 & 1.255557 \\ 1 & -2.736298 & 0.041896 & -1.151967 \\ 6 & -1.150829 & 0.813043 & 0.694083 \\ 1 & -1.948541 & 1.388116 & 1.154513 \\ 1 & -0.259561 & -0.078187 & 2.900139 \\ 1 & 0.798788 & -2.458543 & 1.045106 \\ 1 & -0.939131 & -2.247750 & -1.174788\end{array}$

$1-\mathrm{CH}_{2} 8,10-\mathrm{C}_{2} \mathrm{~B}_{10} \mathrm{H}_{11}{ }^{+}(5)$

$\begin{array}{rrrr}5 & -0.550218 & 1.475785 & 0.226965 \\ 1 & 3.011534 & -0.000231 & 0.766261 \\ 6 & -2.785789 & 0.000000 & -0.149285 \\ 5 & -0.116833 & -0.915925 & -1.393597 \\ 5 & -0.551400 & -1.474402 & 0.230975 \\ 5 & -0.115867 & 0.912308 & -1.396251 \\ 5 & -0.923481 & 0.002299 & 1.247590 \\ 5 & 0.630560 & 0.908059 & 1.400680 \\ 5 & 0.629589 & -0.904527 & 1.403263 \\ 5 & 1.390714 & -0.002330 & -1.243322 \\ 6 & 1.065905 & 1.313097 & -0.211032 \\ 6 & 1.064959 & -1.314271 & -0.207403 \\ 1 & -1.690488 & 0.004130 & 2.156579 \\ 1 & -1.092641 & 2.517679 & 0.355265 \\ 1 & -0.302787 & 1.586780 & -2.346699 \\ 1 & -0.304294 & -1.592829 & -2.342209 \\ 1 & -1.094968 & -2.515369 & 0.361930 \\ 1 & 1.645025 & -2.217892 & -0.363947\end{array}$




$\begin{array}{rrrr}1 & 2.259493 & -0.003739 & -2.039341 \\ 1 & 1.646880 & 2.215685 & -0.370130 \\ 1 & 0.941696 & -1.581467 & 2.318180 \\ 1 & 0.943431 & 1.587351 & 2.313595 \\ 5 & -1.325572 & 0.000315 & -0.487610 \\ 5 & 1.862085 & 0.000000 & 0.506364 \\ 1 & -3.340328 & -0.915616 & 0.060762 \\ 1 & -3.340891 & 0.915154 & 0.060795\end{array}$

\section{TS(5-6)}

$\begin{array}{lrrr}1 & 1.497384 & -2.063423 & 1.310243 \\ 1 & -1.176331 & -0.648150 & -2.655012 \\ 6 & -0.618988 & -0.842655 & 1.153757 \\ 5 & 0.379287 & 0.482436 & 1.565408 \\ 1 & -2.882047 & -1.010531 & -0.067264 \\ 1 & 2.979433 & -0.872472 & 0.181736 \\ 5 & -0.767094 & -0.437023 & -1.567381 \\ 5 & 0.247684 & 1.017920 & -1.273573 \\ 5 & 0.974912 & -1.197340 & 0.706771 \\ 5 & -1.765102 & -0.638785 & -0.104585 \\ 1 & -2.219626 & 1.017016 & 1.731196 \\ 1 & -0.631034 & -2.890381 & -0.420597 \\ 1 & -2.079996 & 1.538144 & -1.050391 \\ 1 & 0.500351 & 1.680901 & -2.220512 \\ 1 & -0.155532 & 2.848963 & 0.497961 \\ 6 & -1.292381 & 0.915378 & -0.638742 \\ 5 & -1.324561 & 0.661914 & 1.051687 \\ 1 & 0.591949 & 0.638568 & 2.717285 \\ 6 & 2.164338 & -0.319592 & -0.287114 \\ 1 & -0.909029 & -1.479332 & 1.985399 \\ 5 & -0.074857 & 1.680525 & 0.346665 \\ 5 & -0.410810 & -1.731871 & -0.357421 \\ 5 & 0.813966 & -0.722643 & -0.996163 \\ 1 & 2.277208 & 2.046254 & 0.316853 \\ 1 & 2.428796 & -0.379130 & -1.400265 \\ 5 & 1.578707 & 1.095825 & 0.169783\end{array}$

$$
2,9,11-\mathrm{C}_{3} \mathrm{~B}_{10} \mathrm{H}_{13}{ }^{+}(\mathbf{6})
$$$$
\begin{aligned}
& 1 \\
& 1 \\
& 6 \\
& 5 \\
& 1 \\
& 1 \\
& 5 \\
& 5 \\
& 5 \\
& 5 \\
& 1 \\
& 1 \\
& 1 \\
& 1 \\
& 1 \\
& 6
\end{aligned}
$$

$$
\begin{array}{rrr}
1.315737 & -0.003022 & -2.441525 \\
-0.391444 & 2.432143 & 1.617123 \\
-0.884528 & -0.000054 & -1.282963 \\
-0.277534 & -1.523198 & -0.871703 \\
-2.717238 & 1.345343 & -0.000715 \\
2.975388 & -0.001978 & -0.000006 \\
-0.275600 & 1.523517 & 0.871541 \\
0.837516 & -0.000145 & 1.364355 \\
0.837504 & -0.002567 & -1.364356 \\
-1.629715 & 0.892426 & -0.000449 \\
-2.718773 & -1.341820 & 0.000727 \\
-0.390880 & 2.429893 & -1.620347 \\
-1.370312 & 0.002383 & 2.254676 \\
1.315748 & 0.000076 & 2.441525 \\
-0.394852 & -2.429487 & 1.620287 \\
-0.884518 & 0.001409 & 1.282966
\end{array}
$$

$\mathrm{B}_{6} \mathrm{H}_{5} \mathrm{CH}_{2}^{-}(7)$

$\begin{array}{rrrr}5 & -1.111798 & 0.470483 & -0.892343 \\ 1 & -2.162056 & -0.300745 & 1.575139 \\ 5 & 0.418245 & -0.464292 & 0.878089 \\ 5 & -0.640965 & -1.157243 & -0.417301 \\ 5 & -0.346219 & 1.198451 & 0.411841 \\ 5 & -1.314601 & -0.099222 & 0.747928 \\ 5 & 0.532223 & 0.007070 & -0.792589 \\ 1 & -1.749349 & 0.911350 & -1.808841 \\ 6 & 1.881168 & 0.066747 & -0.202246 \\ 1 & 0.892131 & -0.992625 & 1.840859 \\ 1 & -0.000422 & 2.289609 & 0.760717 \\ 1 & -0.887288 & -2.279098 & -0.762895 \\ 1 & 2.387104 & 0.993015 & 0.083007 \\ 1 & 2.548451 & -0.798226 & -0.152630\end{array}$

\section{TS(7-8)}

$\begin{array}{lrrr}6 & -1.510085 & -0.092147 & -0.094605 \\ 5 & -0.178832 & -1.237990 & 0.371394 \\ 1 & -0.517564 & -2.382442 & 0.496296 \\ 1 & 2.163899 & -1.437870 & -0.688911 \\ 1 & -2.304592 & -0.665998 & 0.376479 \\ 5 & -0.578264 & 1.133603 & 0.399872 \\ 1 & -0.991459 & 2.268731 & 0.496854 \\ 5 & 0.944040 & 0.976315 & -0.474933 \\ 5 & 1.236787 & -0.722495 & -0.458523 \\ 1 & 1.595975 & 1.930317 & -0.778176 \\ 5 & -0.207884 & -0.116798 & -0.975433 \\ 1 & 1.535380 & 0.196760 & 1.958828 \\ 1 & -1.850007 & 0.015036 & -1.227318 \\ 5 & 0.669929 & 0.093033 & 1.124339\end{array}$

$\mathrm{B}_{11} \mathrm{H}_{10} \mathrm{CH}_{2}{ }^{-}(\mathbf{9})$

$\begin{array}{rrrr}5 & 1.602703 & 0.321029 & -0.933555 \\ 5 & 0.334530 & -1.389771 & 0.645966 \\ 5 & -0.699873 & 1.359898 & 0.570668 \\ 5 & 0.220624 & 0.203523 & 1.554097 \\ 5 & -1.129257 & -0.399891 & 0.662758 \\ 5 & 1.486871 & -1.322868 & -0.580377 \\ 5 & 0.039862 & 1.206170 & -1.023149 \\ 5 & 1.761677 & -0.273596 & 0.768530 \\ 5 & 0.023673 & -0.678897 & -1.195531\end{array}$




$\begin{array}{rrrr}5 & -1.502698 & 0.369013 & -0.849763 \\ 5 & 1.040135 & 1.346942 & 0.439117 \\ 1 & 0.106162 & -2.454092 & 1.148235 \\ 1 & -1.344524 & 2.256847 & 1.028734 \\ 1 & 0.138752 & 0.234073 & 2.747287 \\ 1 & 2.112238 & -2.240792 & -1.014908 \\ 1 & -0.025044 & 1.994144 & -1.917635 \\ 1 & 2.743040 & -0.383294 & 1.445174 \\ 1 & -0.381487 & -1.319015 & -2.125358 \\ 1 & -2.195600 & 0.818593 & -1.706440 \\ 1 & 1.641932 & 2.335477 & 0.741289 \\ 1 & 2.413316 & 0.731089 & -1.713293 \\ 6 & -2.495982 & -0.667166 & 0.157392 \\ 1 & -3.358468 & -0.068911 & 0.451219 \\ 1 & -2.765655 & -1.608886 & -0.322450\end{array}$

\section{TS(9-10)}

$\begin{array}{rrrr}5 & 1.217715 & 1.120580 & -0.381904 \\ 5 & 0.380450 & 1.023619 & 1.336221 \\ 5 & -0.106407 & -0.762051 & 1.565917 \\ 5 & -1.290218 & -1.139915 & 0.227049 \\ 1 & 0.669774 & 1.772037 & 2.219999 \\ 1 & 2.139982 & -0.972460 & -1.400734 \\ 5 & 1.292244 & -0.270112 & 0.807629 \\ 1 & 1.995733 & 2.033701 & -0.391068 \\ 5 & -0.162986 & 0.651932 & -1.614522 \\ 1 & 2.867993 & -0.407609 & 0.168175 \\ 6 & 1.924718 & -0.361017 & -0.537485 \\ 1 & -0.718897 & 2.749142 & -0.183808 \\ 1 & -0.067539 & 1.210271 & -2.674491 \\ 5 & -0.511580 & 1.569805 & -0.178066 \\ 5 & -0.620733 & -0.969570 & -1.370586 \\ 5 & -1.643789 & 0.312935 & -0.688271 \\ 5 & 0.458671 & -1.577953 & -0.031060 \\ 1 & -2.031337 & -2.035379 & 0.519024 \\ 1 & -2.758134 & 0.577147 & -1.044245 \\ 5 & -1.276953 & 0.473951 & 1.074615 \\ 1 & -2.132629 & 0.834725 & 1.829649 \\ 1 & -0.123476 & -1.335004 & 2.613626 \\ 1 & 0.901923 & -2.684661 & 0.056461 \\ 1 & -0.973768 & -1.741918 & -2.222782 \\ & & & \\ \left.\mathrm{~B}_{12} \mathrm{H}_{11} \mathrm{CH}-111\right) & & \\ & & & \\ 1 & 0.532370 & -1.500448 & 2.458200 \\ 1 & -1.256717 & 2.479626 & 0.186612 \\ 5 & 1.794725 & 0.000725 & 0.703732 \\ 5 & 1.186396 & -1.455702 & -0.118410 \\ 1 & 1.832933 & 2.457285 & -0.211051 \\ 5 & -1.238252 & -0.001157 & -0.712758 \\ 5 & -0.550721 & 1.516564 & 0.117583 \\ 5 & -0.549824 & -1.516868 & 0.118293 \\ 5 & 0.444784 & -0.924326 & 1.414674 \\ 5 & 1.185401 & 1.456054 & -0.119390 \\ 1 & 2.473928 & 0.000322 & -1.858257\end{array}$

$\begin{array}{rrrr}1 & 0.531767 & 1.502360 & 2.457404 \\ 1 & -1.255198 & -2.480288 & 0.189014 \\ 1 & -1.872198 & -0.000691 & 1.992772 \\ 1 & -0.107275 & -1.525226 & -2.438616 \\ 5 & 0.076241 & 0.927817 & -1.418935 \\ 5 & -1.093634 & 0.000211 & 1.085556 \\ 1 & 1.834680 & -2.456543 & -0.208880 \\ 6 & -2.633016 & -0.000244 & -0.214495 \\ 1 & 2.865462 & 0.001333 & 1.237793 \\ 5 & 0.077228 & -0.929019 & -1.418422 \\ 5 & 0.444270 & 0.925596 & 1.414223 \\ 1 & -0.108144 & 1.523701 & -2.439329 \\ 5 & 1.564690 & 0.000000 & -1.080800 \\ 1 & -3.190150 & -0.911706 & -0.001569 \\ 1 & -3.189887 & 0.911865 & -0.003858\end{array}$

\section{TS(11-12)}

$\begin{array}{rrrr}1 & 1.534629 & -2.162017 & 1.128360 \\ 1 & -1.112680 & -0.543702 & -2.720260 \\ 5 & -0.708349 & -0.997034 & 1.226831 \\ 5 & 0.397876 & 0.365679 & 1.579312 \\ 1 & -2.919308 & -1.050842 & -0.204731 \\ 1 & 2.984630 & -0.890474 & 0.097490 \\ 5 & -0.790047 & -0.327637 & -1.590282 \\ 5 & 0.240994 & 1.085155 & -1.210493 \\ 5 & 0.955711 & -1.227802 & 0.668657 \\ 5 & -1.805473 & -0.622780 & -0.134049 \\ 1 & -2.181521 & 1.007054 & 1.855649 \\ 1 & -0.582730 & -2.871250 & -0.675488 \\ 1 & -2.259795 & 1.787569 & -1.061105 \\ 1 & 0.656018 & 1.733342 & -2.129483 \\ 1 & -0.052888 & 2.812003 & 0.729794 \\ 5 & -1.408804 & 1.065756 & -0.630778 \\ 5 & -1.354049 & 0.607441 & 1.090061 \\ 1 & 0.687906 & 0.555536 & 2.726233 \\ 6 & 2.155917 & -0.302169 & -0.301029 \\ 1 & -0.966101 & -1.776068 & 2.096732 \\ 5 & -0.097538 & 1.652850 & 0.440162 \\ 5 & -0.438790 & -1.709622 & -0.437409 \\ 5 & 0.767446 & -0.665650 & -1.002065 \\ 1 & 2.300920 & 2.036315 & 0.353690 \\ 1 & 2.330904 & -0.396122 & -1.445471 \\ 5 & 1.569925 & 1.087976 & 0.211007\end{array}$

$\mathrm{B}_{12} \mathrm{CH}_{13}{ }^{-}(12)$

$\begin{array}{rrrr}1 & 1.405110 & -0.008224 & -2.415442 \\ 1 & -0.323507 & 2.503233 & 1.543908 \\ 5 & -0.998871 & -0.001322 & -1.420225 \\ 5 & -0.285600 & -1.510027 & -0.877643 \\ 1 & -2.702193 & 1.473097 & -0.002838 \\ 1 & 2.981623 & -0.005330 & 0.000040 \\ 5 & -0.280914 & 1.510751 & 0.877611 \\ 5 & 0.817235 & 0.001343 & 1.377589 \\ 5 & 0.817164 & -0.005952 & -1.377638\end{array}$




$\begin{array}{lrrr}5 & -1.656560 & 0.893956 & -0.001839 \\ 1 & -2.706629 & -1.464717 & 0.002990 \\ 1 & -0.321640 & 2.495760 & -1.555152 \\ 1 & -1.503946 & 0.007358 & 2.504430 \\ 1 & 1.405717 & 0.001860 & 2.415095 \\ 1 & -0.330072 & -2.494879 & 1.555207 \\ 5 & -0.998698 & 0.004395 & 1.420487 \\ 5 & -1.659221 & -0.888797 & 0.001761 \\ 1 & -0.332186 & -2.502428 & -1.543776 \\ 6 & 1.895287 & -0.002038 & -0.000062 \\ 1 & -1.504434 & -0.002573 & -2.504033 \\ 5 & -0.285095 & -1.505778 & 0.884087 \\ 5 & -0.280728 & 1.506585 & -0.883852 \\ 5 & 1.263384 & 1.387156 & -0.001173 \\ 1 & 2.000695 & -2.327420 & 0.001664 \\ 1 & 2.007895 & 2.323292 & -0.002170 \\ 5 & 1.258272 & -1.389671 & 0.000926 \\ 2-\mathrm{CH}_{2}-1,7-\mathrm{C}_{2} \mathrm{~B}_{9} \mathrm{H}_{10}{ }^{+}(\mathbf{1 3}) & \end{array}$

$\begin{array}{rrrr}5 & 1.167555 & -0.000055 & -0.184945 \\ 5 & -1.148294 & 1.478102 & 0.242435 \\ 5 & -1.148387 & -1.477872 & 0.242346 \\ 5 & -1.701779 & 0.000078 & -0.604150 \\ 5 & -1.270292 & -0.000005 & 1.194480 \\ 6 & 0.463294 & 1.508798 & -0.218715 \\ 6 & 0.463106 & -1.508856 & -0.218701 \\ 5 & -0.463910 & 0.996263 & -1.370439 \\ 5 & 0.239737 & 0.952029 & 1.266406 \\ 5 & 0.239597 & -0.952155 & 1.266515 \\ 5 & -0.464055 & -0.996209 & -1.370590 \\ 1 & -1.759181 & 2.478346 & 0.381804 \\ 1 & -1.759344 & -2.478125 & 0.381528 \\ 1 & -2.796196 & 0.000056 & -1.078191 \\ 1 & -2.014016 & 0.000110 & 2.122848 \\ 1 & 1.099129 & 2.377830 & -0.357104 \\ 1 & 1.098658 & -2.378060 & -0.357273 \\ 1 & -0.393618 & 1.391605 & -2.481354 \\ 1 & 0.744253 & 1.461330 & 2.204091 \\ 1 & 0.743953 & -1.461622 & 2.204175 \\ 1 & -0.393755 & -1.391324 & -2.481559 \\ 6 & 2.678720 & -0.000080 & -0.174272 \\ 1 & 3.274230 & -0.913399 & -0.139507 \\ 1 & 3.274299 & 0.913200 & -0.139614\end{array}$

\section{TS(13-14)}

5
5
5
5
5
6
6
5
5
5
$1-\mathrm{CH}_{2}-1,7-\mathrm{C}_{2} \mathrm{~B}_{9} \mathrm{H}_{10}{ }^{+}(15)$

$\begin{array}{rrrr}5 & -0.170011 & -1.374341 & -0.242574 \\ 5 & 1.962267 & 0.333544 & -0.124924 \\ 5 & -0.723872 & 1.226445 & 0.740256 \\ 5 & 0.637306 & 1.512362 & -0.415897 \\ 5 & 0.910076 & 0.805591 & 1.237435 \\ 6 & 1.349252 & -1.093664 & -0.669987 \\ 6 & -1.351282 & -0.209167 & 0.096616 \\ 5 & 0.802362 & 0.093320 & -1.532145 \\ 5 & 1.269498 & -0.944563 & 0.936064 \\ 5 & -0.384245 & -0.409255 & 1.428556 \\ 5 & -0.873148 & 0.891426 & -1.018546 \\ 1 & 3.106062 & 0.596343 & -0.250120 \\ 1 & -1.456515 & 2.006715 & 1.243380 \\ 1 & 0.878305 & 2.597322 & -0.832146 \\ 1 & 1.353965 & 1.385619 & 2.168682 \\ 1 & 1.915861 & -1.900452 & -1.122067 \\ 6 & -2.679870 & -0.468475 & -0.179462 \\ 1 & 0.858920 & 0.056184 & -2.713477 \\ 1 & 1.843153 & -1.691440 & 1.648312 \\ 1 & -0.910876 & -0.876106 & 2.376600 \\ 1 & -1.571476 & 1.319876 & -1.868352 \\ 1 & -0.635631 & -2.421878 & -0.537490 \\ 1 & -3.437251 & 0.301152 & -0.033357 \\ 1 & -3.004282 & -1.418140 & -0.604090\end{array}$

\section{TS(15-14)}

$\begin{array}{lrrr}6 & -1.198345 & -0.659416 & 0.466752 \\ 1 & 0.219368 & 2.773663 & 0.585806 \\ 1 & -0.369383 & -2.572835 & -0.954377 \\ 6 & 0.909863 & -0.428049 & -1.315457 \\ 5 & 1.538750 & -0.924759 & 0.130339 \\ 6 & -1.842644 & -0.371081 & -0.742471 \\ 5 & 0.097837 & -1.170269 & 1.262839 \\ 5 & -0.671205 & 0.501912 & 1.455164 \\ 5 & 1.057273 & 0.348813 & 1.343213 \\ 5 & 0.162334 & 1.008793 & -1.352877 \\ 5 & -1.397852 & 1.103040 & -0.262994 \\ 5 & 1.618810 & 0.764321 & -0.342825\end{array}$




$\begin{array}{lrrr}1 & 0.043196 & -2.053338 & 2.048292 \\ 1 & -2.619846 & -0.829299 & 0.141079 \\ 1 & -2.047415 & -0.981395 & -1.621982 \\ 1 & 1.413276 & -0.767800 & -2.216349 \\ 1 & 2.403495 & -1.727583 & 0.202089 \\ 1 & 2.685867 & 1.207313 & -0.596415 \\ 1 & 0.001847 & 1.570928 & -2.383474 \\ 1 & -2.284573 & 1.832646 & 0.029073 \\ 1 & 1.739203 & 0.633363 & 2.266060 \\ 1 & -1.281910 & 0.849636 & 2.407088 \\ 5 & -0.054032 & -1.500043 & -0.581992 \\ 5 & 0.224813 & 1.631388 & 0.277166\end{array}$

\section{1- $\mathrm{CH}_{2} 2,3-\mathrm{C}_{2} \mathrm{~B}_{4} \mathrm{H}_{5}$}

$\begin{array}{lrrr}6 & 0.917358 & -0.616525 & 0.859000 \\ 1 & 2.812543 & 0.041916 & -0.699764 \\ 5 & 0.335231 & 0.843123 & -0.877796 \\ 6 & 0.327681 & -0.843129 & -0.557760 \\ 5 & 0.467433 & 0.885939 & 0.842139 \\ 5 & 1.699359 & 0.103872 & -0.269579 \\ 5 & -0.971640 & 0.035325 & -0.119667 \\ 1 & 1.164116 & -1.395069 & 1.571013 \\ 6 & -2.393644 & -0.075079 & 0.074849 \\ 1 & 0.186383 & 1.457201 & -1.891557 \\ 1 & 0.568872 & 1.759601 & 1.656727 \\ 1 & 0.459388 & -1.723440 & -1.187022 \\ 1 & -2.981347 & 0.705450 & 0.562306 \\ 1 & -2.970238 & -0.978555 & -0.143728\end{array}$

1- $\mathrm{CH}_{2} 7,9-\mathrm{C}_{2} \mathrm{~B}_{9} \mathrm{H}_{10}{ }^{-}$

$\begin{array}{rrrr}5 & 0.627844 & 1.042345 & -0.818091 \\ 5 & -2.013651 & 0.200749 & 0.174203 \\ 5 & 0.554433 & -0.870532 & 1.029890 \\ 5 & -1.106685 & -1.303540 & 0.577346 \\ 5 & -0.821944 & 0.206458 & 1.471416 \\ 5 & -0.958180 & 0.548087 & -1.334762 \\ 5 & 1.688360 & -0.087217 & -0.033289 \\ 6 & -1.514232 & -0.872175 & -0.915767 \\ 6 & -0.735561 & 1.353825 & 0.187577 \\ 5 & 0.733930 & 0.929141 & 0.976779 \\ 5 & 0.142413 & -0.963034 & -0.869229 \\ 1 & -3.144867 & 0.539108 & 0.323874 \\ 1 & 0.975392 & -1.635360 & 1.850206 \\ 1 & -1.586678 & -2.288609 & 1.051399 \\ 1 & -1.209840 & 0.516592 & 2.556013 \\ 1 & -1.384373 & 1.115085 & -2.293232 \\ 1 & -2.172055 & -1.445615 & -1.554848 \\ 1 & -1.062050 & 2.377236 & 0.329627 \\ 1 & 1.209627 & 1.736273 & 1.713676 \\ 1 & 0.582592 & -1.740206 & -1.655211 \\ 1 & 0.995244 & 1.956904 & -1.497695 \\ 6 & 3.113928 & -0.288477 & -0.256888 \\ 1 & 3.701683 & 0.322745 & -0.946141 \\ 1 & 3.677912 & -1.125464 & 0.161490\end{array}$

1- $\mathrm{CH}_{2} 8,10-\mathrm{C}_{2} \mathrm{~B}_{10} \mathrm{H}_{11}{ }^{-}$

$\begin{array}{rrrr}5 & 0.058218 & 1.571053 & 0.000000 \\ 5 & 0.546057 & 0.442563 & 1.389958 \\ 5 & 1.627286 & 0.258869 & 0.000000 \\ 5 & 0.546057 & 0.442563 & -1.389958 \\ 5 & -1.162159 & 0.557942 & -0.886605 \\ 5 & -1.162159 & 0.557942 & 0.886605 \\ 1 & 0.846492 & 0.795081 & 2.489827 \\ 1 & 2.737434 & 0.695686 & 0.000000 \\ 1 & 0.846492 & 0.795081 & -2.489827 \\ 1 & -2.007106 & 0.994259 & -1.602476 \\ 1 & -2.007106 & 0.994259 & 1.602476 \\ 5 & 1.188050 & -1.182135 & 0.866148 \\ 5 & 1.188050 & -1.182135 & -0.866148 \\ 6 & -0.494432 & -0.951897 & -1.300106 \\ 5 & -1.549007 & -0.919683 & 0.000000 \\ 6 & -0.494432 & -0.951897 & 1.300106 \\ 5 & -0.113548 & -1.996650 & 0.000000 \\ 1 & 1.859904 & -1.834785 & 1.603002 \\ 1 & 1.859904 & -1.834785 & -1.603002 \\ 1 & -0.832849 & -1.418969 & -2.217412 \\ 1 & -2.609624 & -1.456140 & 0.000000 \\ 1 & -0.832849 & -1.418969 & 2.217412 \\ 1 & -0.348684 & -3.160837 & 0.000000 \\ 6 & 0.053078 & 3.043083 & 0.000000 \\ 1 & 0.134236 & 3.631370 & -0.915390 \\ 1 & 0.134236 & 3.631370 & 0.915390\end{array}$

$\mathrm{B}_{6} \mathrm{H}_{5} \mathrm{CH}_{2}{ }^{-3}$

$\begin{array}{rrrr}5 & 0.583116 & -0.054100 & -1.217363 \\ 1 & 2.999596 & -0.000940 & 0.066139 \\ 5 & 0.511259 & 0.053831 & 1.227090 \\ 5 & 0.484417 & 1.215749 & -0.052430 \\ 5 & 0.479882 & -1.215729 & 0.056984 \\ 5 & 1.760457 & -0.000989 & 0.043410 \\ 5 & -0.811820 & 0.000848 & -0.043411 \\ 1 & 0.651866 & -0.111059 & -2.453166 \\ 6 & -2.422740 & 0.000166 & -0.172702 \\ 1 & 0.484616 & 0.110244 & 2.465294 \\ 1 & 0.509005 & -2.455569 & 0.117305 \\ 1 & 0.523589 & 2.455905 & -0.101056 \\ 1 & -2.834171 & -0.870451 & 0.436801 \\ 1 & -2.834617 & 0.872828 & 0.433501 \\ & & & \\ \mathrm{~B}_{11} \mathrm{H}_{10} \mathrm{CH}_{2}-3 & & \\ & & & \\ 5 & 0.000000 & 1.283860 & 0.000000 \\ 5 & 2.046631 & -0.431092 & 0.000000 \\ 5 & -0.798917 & -1.329714 & 0.000000 \\ 5 & 0.726872 & -1.263011 & 0.905610 \\ 5 & 0.726872 & -1.263011 & -0.905610 \\ 5 & 1.749311 & 1.278248 & 0.000000 \\ 5 & -1.680272 & 0.253668 & 0.000000\end{array}$




\begin{tabular}{|c|c|c|c|}
\hline 5 & 1.151091 & 0.435592 & \\
\hline b & 1.151091 & 0.435592 & -1.323953 \\
\hline D & -0.614371 & -0.120398 & \\
\hline D & -0.614371 & -0.120398 & \\
\hline 1 & 3.170523 & -0.911300 & 0.0 \\
\hline 1 & -1.454602 & -2.354051 & \\
\hline & 1.021441 & -2.204981 & 1.6233 \\
\hline & 1.021441 & -2.204981 & -1.623 \\
\hline & 2.526767 & 2.218691 & 0.0 \\
\hline & -3.144409 & 0.645770 & \\
\hline & 1.5242 & 0.709560 & \\
\hline 1 & 1.524245 & 0.709560 & -2.455 \\
\hline & -1.035271 & -0.130951 & -2.46 \\
\hline | & -1.035271 & -0.130951 & \\
\hline & -0.417193 & 2.4 & 0.000 \\
\hline & -3.5 & 1.1 & \\
\hline & -3.599782 & 1.10 & -0 \\
\hline \multicolumn{4}{|c|}{$\mathrm{B}_{12} \mathrm{H}_{11} \mathrm{CH}_{2}^{-3}$} \\
\hline & -1.709432 & 0.796945 & -2.4420 \\
\hline & 0.974183 & 2.114904 & 1.508240 \\
\hline & -2.0432 & -0.00 & 0.04 \\
\hline 5 & -1.105844 & -1.22 & -0.870810 \\
\hline & -1.597578 & 0.816518 & 2.502174 \\
\hline 5 & 1.529479 & 0.002320 & -0.05560 \\
\hline 5 & 0.465790 & 1.21 & 0.869181 \\
\hline 5 & 0.397272 & -0.467463 & -1.464450 \\
\hline 5 & -1.134819 & 0.46 & -1.42 \\
\hline 5 & -1.068269 & 0.46 & 1.46 \\
\hline 1 & -1.598732 & -2.1114 & 1.56165 \\
\hline 1 & -1.626202 & 2.607313 & 0.026037 \\
\hline 1 & 0.868808 & -0.815403 & -2.526499 \\
\hline 1 & 0.896304 & 2.114059 & -1.578864 \\
\hline & 0.946378 & -2.609657 & -0.016077 \\
\hline 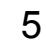 & 0.462032 & -0.46 & 1.43 \\
\hline 5 & 0.420720 & 1.215237 & -0.914607 \\
\hline 1 & -1.667084 & -2.116223 & -1.488705 \\
\hline 6 & 3.102207 & 0.008483 & -0.147542 \\
\hline 1 & -3.260623 & -0.002389 & 0.068499 \\
\hline & 0.454147 & -1.497313 & -0.013625 \\
\hline & -1.082254 & 1.5171 & 0.015811 \\
\hline & 0.991445 & -0.799485 & 2.470945 \\
\hline & -1.068297 & -1.222148 & 0.918810 \\
\hline & 3.518899 & -0.903787 & 0.354645 \\
\hline & 3.516999 & 0.856237 & 0.458469 \\
\hline
\end{tabular}

